Cahiers $d u$ MONDE RUSSE

\section{Cahiers du monde russe}

Russie - Empire russe - Union soviétique et États indépendants

$58 / 4 \mid 2017$

Varia

Tatyana V. Bakhmetyeva, Mother of the Church: Sofia Svechina, the Salon and the Politics of Catholicism in Nineteenth-Century Russia and France

\title{
Laura Pettinaroli
}

\section{(2) OpenEdition Journals \\ Édition électronique \\ URL : http://journals.openedition.org/monderusse/10154 \\ DOI : 10.4000/monderusse.10154 \\ ISSN : $1777-5388$ \\ Éditeur \\ Éditions de l'EHESS}

\section{Édition imprimée}

Date de publication : 1 octobre 2017

Pagination : 694-696

ISBN : 978-2-7132-2698-4

ISSN : $1252-6576$

Référence électronique

Laura Pettinaroli, «Tatyana V. Bakhmetyeva, Mother of the Church: Sofia Svechina, the Salon and the Politics of Catholicism in Nineteenth-Century Russia and France ", Cahiers du monde russe [En ligne], 58/4 | 2017, mis en ligne le 01 octobre 2017, consulté le 06 janvier 2021. URL : http:// journals.openedition.org/monderusse/10154; DOI : https://doi.org/10.4000/monderusse.10154 
Tatyana V. BAKHMETYEVA

\section{Mother of the Church}

Sofia Svechina, the Salon and the Politics of Catholicism in Nineteenth-Century Russia and France

DeKalb : Northern Illinois University Press, 2016, 332 p.

Tatyana V. Bakhmetyeva, enseignante au Susan B. Anthony Institute de l'université de Rochester, NY, livre ici une biographie de Sophie Swetchine (1782-1857), une femme de l'aristocratie russe, née Soymonov, élevée dans l'esprit des Lumières mais qui évolue vers la pratique religieuse au temps des guerres napoléoniennes. Elle se tourne d'abord vers l'orthodoxie avant d'embrasser le catholicisme en 1814, au terme d'une longue quête intellectuelle et de nombreux échanges avec Joseph de Maistre. Elle s'installe à Paris en 1816, où elle tient un salon à partir de 1825 qui accueille de brillantes figures du catholicisme (Lacordaire, Falloux, Montalembert, Guéranger...). Pour étudier cette figure déjà bien connue, l'auteure se fonde sur des archives essentiellement françaises - celles de la Bibliothèque slave des jésuites alors à Meudon (fonds Gagarin, un parent de S. Swetchine qui devient 
jésuite), quelques dossiers de correspondance (avec Lacordaire aux Archives nationales, avec Dom Guéranger à l'abbaye de Solesmes) — auxquelles s'ajoutent les mémoires de Praskovija Golovina conservés dans les archives historiques de la Fédération de Russie. Cependant l'étude est surtout tributaire de sources imprimées, comme la correspondance de Swetchine publiée par Falloux à la fin du XIX ${ }^{\mathrm{e}}$ siècle.

La question qui se pose d'emblée face à ce parcours bien balisé par les nombreuses biographies de Madame Swetchine est la suivante : comment proposer une approche neuve, et non hagiographique, de ce personnage féminin du premier XIX ${ }^{\mathrm{e}}$ siècle ? La démarche de Bakhmetyeva se déploie à différents niveaux. Elle engage d'abord une approche résolument réflexive : la conclusion, très opportunément intitulée « Writing the Modern Saint » (p. 260-271), souligne les différentes phases d'investissements de la figure de Mme Swetchine : par les catholiques libéraux puis, plus largement, par des catholiques en quête de modèles de femmes et de laïcs engagés, mais aussi par des auteurs protestants qui mettent en valeur le parcours de cette convertie. Les perspectives suggérées ici sont fécondes et mériteraient d'être poursuivies pour le $\mathrm{XX}^{\mathrm{e}}$ siècle (notamment autour du p. Rouët de Journel qui fait de Swetchine un modèle pour les catholiques russes) afin de préciser la chronologie et les tonalités, assez variées, de ces accès de dévotions et d'intérêt, mais aussi de désintérêt, pour Mme Swetchine. Cependant, au-delà de cette approche réflexive, c'est surtout en privilégiant deux axes de lecture que Bakhmetyeva tente, et parvient, à renouveler notre perspective sur Sophie Swetchine. En effet, si la vie de Sophie Swetchine est bien connue en France, notamment des historiens du religieux, elle l'est moins des spécialistes d'histoire russe ou d'histoire du genre. Sous ces deux points de vue, l'ouvrage de Tatyana Bakhmetyeva offre un indéniable apport.

C'est sans doute sur la dimension russe de Mme Swetchine que le lecteur apprend le plus : l'ouvrage, structuré en deux périodes (Saint-Pétersbourg, p. 11-108 ; Paris, p. 109-260), met bien en perspective l'éducation de la jeune Sophie dans un milieu acquis aux Lumières, la crise-tournant de 1812 (chapitres 1 et 2), les questionnements de toute une génération sur l'identité russe et le rapport à l'Occident, la première phase du débat slavophiles/occidentalistes (chapitres 3 et 4 ). Cependant ce fil russe de la vie de Madame Swetchine est également déroulé au cours des années parisiennes (et des escapades romaines), par l'étude de la correspondance avec des membres de la noblesse russe ou encore les réflexions qu'inspirent à Swetchine les évolutions politiques et religieuses de son pays natal. Ce faisant, Bakhmetyeva éclaire bien certains paradoxes, comme la condamnation par cette proche des catholiques libéraux des Décabristes et des révoltes polonaises de 1830 : en effet, ces réprobations sont surtout exprimées à des correspondants russes, proches du pouvoir impérial, avec lequel Swetchine doit maintenir l'entente pour conserver ses biens en Russie malgré sa résidence à l'étranger. Au-delà cependant de ces intérêts bien compris, l'auteure s'emploie à traquer les traces d'un attachement affectif au pays d'origine et s'interroge sur la réception en Russie de la figure de Swetchine, très négative à l'heure de sa mort en 1857, aux lendemains de guerre de Crimée. Sur ce versant russe, on pourra cependant regretter que la bibliographie italienne ne soit guère prise en compte, notamment les études de Sabina Pavone sur les jésuites 
et celles d'Angelo Tamborra sur les milieux russes en Italie au XIX ${ }^{\mathrm{e}}$ siècle. De toute évidence, cette perspective russe sur Swetchine ouvre des pistes nouvelles et on ne peut qu'espérer qu'elles soient poursuivies, par exemple par une interrogation des sources diplomatiques (Bakhmetyeva rappelle notamment que Madame Swetchine séjourne à Rome en 1825 auprès de l'ambassade de Russie).

Cependant cet ouvrage revisite aussi la vie de Madame Swetchine sous un second angle : l'histoire du genre, en mettant l'accent sur le salon comme lieu de sociabilité où les femmes jouent un rôle de médiation au seuil du politique. L'auteure intègre ici l'apport de l'historiographie anglophone et francophone pour souligner l'importance des salons dans la période de la Restauration et de la Monarchie de Juillet, comme antichambres des assemblées (plus ou moins) représentatives. Bakhmetyeva met bien en lumière la genèse du salon de Swetchine, notamment la phase préparatoire où la dame russe se forme auprès de salonnières comme Juliette Récamier, avant de prendre son indépendance et de créer un lieu différent par sa tonalité religieuse (chapitres 5 et 6). L'ambiance du salon de Madame Swetchine, y compris dans des détails matériels, est bien rendue, de même que ses principaux acteurs. L'auteure défend la thèse que « the mystery of her appeal » (p. 3) réside dans la capacité particulière de Madame Swetchine à faire s'exprimer et à accueillir en son salon des sensibilités différentes. Cependant, si on voit bien l'importance de Swetchine dans la relation de tête-à-tête avec telle ou telle personnalité ( « she was a confessor », nous dit l'auteure p. 145, une approche qui pourrait être creusée dans une perspective genrée à la lumière de travaux en cours comme ceux de Caroline Muller), on saisit moins ce qu'apporte la sociabilité du salon aux fameux binômes formés par Swetchine, qu'il s'agisse de Lacordaire, Falloux ou Tocqueville. Sans doute fallait-il, pour répondre à cette dernière question, envisager des sources plus larges, embrassant l'ensemble de la correspondance des acteurs du salon, afin de mieux faire apparaître Swetchine comme «tiers ». Certains exemples sont pourtant connus, comme la rencontre, au sein du salon Swetchine, entre Lacordaire et la comtesse de Mesnard, qui joua ensuite un rôle clé dans le développement des tertiaires dominicaines. Plus largement, les analyses de réseaux déployés sur les milieux catholiques du premier $\mathrm{XIX}^{\mathrm{e}}$ siècle par des historiens comme Matthieu Brejon de Lavergnée (Société de Saint-Vincent de Paul) auraient ici pu offrir un précieux contrepoint, d'autant que les sources disponibles sont vastes et ne sont qu'en partie utilisées (plusieurs fonds ne sont pas sollicités comme les archives des dominicains de la Province de France, les archives jésuites à Vanves, celles de l'archevêché de Paris, mais aussi les papiers Montalembert et surtout Falloux à la Bibliothèque nationale qui comprennent de nombreuses lettres de Madame Swetchine).

Pour conclure, on lira avec intérêt cette étude qui ouvre bien des pistes et permet de revisiter une figure, que l'on croyait bien connaître, mais sur laquelle il reste à l'évidence encore beaucoup à faire.

\section{Laura Pettinaroli}

